\title{
RELATOS DAS AÇÕES \\ EXTENSIONISTAS DA LIGA \\ ACADÊMICA DO TRATO \\ GASTROINTESTINAL E \\ DOENÇAS METABÓLICAS
}

\author{
REPORTS OF THE OUTREACH \\ ACTIONS OF THE ACADEMIC \\ LEAGUE OF GASTROINTESTINAL \\ TRACT AND METABOLIC DISEASES
}

Eduarda Mirela da Silva Montiel"

Matheo Augusto Morandi Stumpf*

Jefferson Matsuiti Okamoto ${ }^{* * *}$

Marcos Ricardo da Silva Rodrigues ${ }^{* * * *}$

Cíbele Pereira Kopruszynski*

Gianna Carla Alberti Schrut ${ }^{* * * * *}$

\section{RESUMO}

As ligas acadêmicas na área da saúde, como projeto extensionista, desenvolvem atividades extracurriculares voltadas para a educação médica, pesquisa científica e promoção da saúde. Essa forma de extensão acadêmica, principalmente no curso de Medicina, fornece oportunidade de crescimento pessoal e profissional aos seus integrantes, apresentando efeito positivo na formação dos mesmos. Por esse motivo, este artigo tem como objetivo relatar as experiências que a "Liga Acadêmica do Trato Gastrointestinal e Doenças Metabólicas", desenvolvida por alunos e professores de Medicina da Universidade Estadual de Ponta Grossa (UEPG), teve em seu primeiro ano como projeto extensionista, tendo em vista a análise e compreensão de seu papel na formação dos acadêmicos, sua inserção como um importante agente de atenção à saúde no município e sua atuação na comunidade, por meio das atividades realizadas. Com isso, destacam-se a importância de ligas acadêmicas para a formação de profissionais qualificados, além da influência positiva gerada para a comunidade.

Palavras-chave: Relações Instituição-Comunidade; Trato Gastrointestinal; Doenças Metabólicas.

\footnotetext{
* Aluna de graduação da Universidade Estadual de Ponta Grossa (UEPG), PR - Brasil. E-mail: eduarda.montiel@gmail.com

** Aluno de graduação da Universidade Estadual de Ponta Grossa (UEPG), PR - Brasil. E-mail: matheoaugusto@hotmail.com

*** Aluno de graduação da Universidade Estadual de Ponta Grossa (UEPG), PR - Brasil. E-mail: okamotojeff@gmail.com

**** Professor da Universidade Estadual de Ponta Grossa (UEPG), PR - Brasil. E-mail: marcos.rrod@uol.com.br

***** Professora da Universidade Federal do Paraná (UFPR), PR - Brasil. E-mail: cibelek@uol.com.br

****** Professora da Universidade Estadual de Ponta Grossa (UEPG), PR - Brasil. E-mail: gialberti@uol.com.br
} 


\section{ABSTRACT}

In health area, academic leagues, as outreach project, develop extracurricular activities for medical education, scientific research and health promotion. It provides, especially in Medicine, opportunity for personal and professional improvement, and has a positive effect on their education. For this reason, this article aims to report the experiences of "The Academic League of Gastrointestinal Tract and Metabolic Diseases" during the first year of its existence as an outreach project, analyzing and understanding, through the activities developed, its role in students' education, its inclusion as an important health care agent in the city and its role in the community. Thus, it highlights the importance of academic leagues to the education of health care professional as well as having a positive influence on community.

\section{Keywords: Community-Institutional Relations; Gastrointestinal Tract; Metabolic Diseases.}

\section{Introduçáo}

Ligas acadêmicas relacionadas à saúde podem ser definidas como organizaçóes estudantis sem fins lucrativos que criam para seus membros oportunidades de atividades didáticas, científicas, culturais e sociais, abrangendo uma determinada área da saúde, visando seu aprendizado e desenvolvimento, sendo geridas pelos próprios estudantes, sob orientação de docentes (PÊGO-FERNANDES et al., 2011; HAMAMOTO FILHO et al., 2011; COSTA et al., 2009; MONTEIRO et al., 2008; NEVES et al., 2009).

Elas atuam como projeto extensionista dentro da universidade e, dessa forma, representam uma oportunidade singular para o desenvolvimento de atividades extracurriculares, direcionadas para a educação médica, pesquisa científica e promoção da saúde, que, quando corretamente direcionadas, colaboram positivamente com a formaçấo de seus participantes (PÊGO-FERNANDES et al., 2011; FERREIRA et al., 2011; MONTEIRO et al., 2008; ANDRADE et al., 2007).

Os primeiros relatos referentes a este tipo de atividades ocorreram na segunda metade do século XIX, nos quais destacam-se a participação de acadêmicos em campanhas de saúde na Inglaterra e a realização de atividades focadas na prestaçắo de serviços em áreas rurais e urbanas por acadêmicos de universidades americanas (FREITAS JÚNIOR et al., 2014; HAMAMOTO FILHO, 2011). A extensão universitária, como uma das funçóes que compóem os pilares da universidade, possui cunho social e envolve relaçôes interdisciplinares entre as práticas de ensino e pesquisa, tornando-se parte orgânica do currículo na formação de educadores e profissionais (MONTEIRO et al., 2008; JEZINE, 2004).

A institucionalização de uma extensão verdadeiramente acadêmica exige uma intensa articulação interna e externa às universidades, referente à formulação de uma política pedagógica que permita a interação entre extensão, ensino e pesquisa, à formulação de parcerias de dimensão interinstitucional e à integração com a comunidade (JEZINE, 2004).

Os programas de extensão universitária desvelam a importância de sua existência na relação estabelecida entre instituição e sociedade, consolidando-se através da aproximação, troca de conhecimentos e experiências entre docentes, discentes e população. $\mathrm{Na}$ área da saúde, assumem particular importância na medida em que se integram à rede assistencial e podem servir de espaço diferenciado para novas experiências voltadas à humanização, ao cuidado e à qualificação da atenção à saúde (BASTOS et al., 2012; HAMAMOTO FILHO et al., 2010; HENNINGTON, 2005). Assim, criaram-se as ligas acadêmicas, com o objetivo de relacionar os diversos saberes para a produçáo do conhecimento com a realidade social (SANTANA, 2012; FERREIRA et al., 2011; JEZINE, 2004).

Ao refletir sobre esses conceitos, denota-se o aprimoramento do ensino e a formação de profissionais como sujeitos ativos, com uma visáo ampliada da saúde, respondendo às necessidades da populaçáo, que 
viabiliza a relação transformadora entre a universidade e a sociedade (FREITAS JÚNIOR et al., 2014; SANTANA, 2012; FERREIRA et al., 2011; HAMAMOTO FILHO et al., 2010).

No curso de Medicina, alguns autores (PÊGO-FERNANDES et al., 2011; HAMAMOTO FILHO, 2011) apontam pontos negativos, como a controvérsia de colaboração ou prejuízo ao ensino no período da graduação, em vista de que muitos alunos acabam por dar maior importância às atividades extracurriculares em detrimento daquelas próprias do curso. No entanto, é fato que as ligas representam uma oportunidade para o aprendizado, que acaba por ocorrer de uma forma dinâmica, já que as atividades são desenvolvidas pelos próprios discentes (PÊGO-FERNANDES et al., 2011; VIERA et al., 2004).

No conjunto das atividades de uma liga acadêmica de Medicina, estão as atividades teóricas, como aulas, seminários, discussóes de textos, apresentaçôes de casos clínicos, ou práticas, como por exemplo o atendimento a pacientes e desenvolvimento de projetos científicos. Além de atividades educativas para orientação da população, participação em programas comunitários, desenvolvimento de campanhas de saúde, entre outras, que são importantes atividades sociais (PÊGO-FERNANDES et al., 2011; HAMAMOTO FILHO et al., 2010).

Nesse sentido, o estatuto de uma liga acadêmica deve prever quais são seus objetivos, as obrigaçóes dos membros, a formação da diretoria e descrever suas atividades. Deve também definir seus participantes, especificando quais são os acadêmicos (de que cursos, anos e instituiçóes) que podem fazer parte e quantas vagas estáo disponíveis (BASTOS et al., 2012; PÊGO-FERNANDES et al., 2011).

Assim, a conformação da Liga Acadêmica do Trato Gastrointestinal e Doenças Metabólicas (LATGIDM) decorreu da percepção de que o padrão alimentar atual favorece o desenvolvimento de doenças metabólicas (MELLO et al., 2009). O processo de refinamento de grãos aumenta seu índice glicêmico, reduz a quantidade de fibras em $80 \%$ e de proteínas em $30 \%$, restando compostos ricos em carboidratos de alta absorçáo. A substituição de uma dieta rica em fibras e de baixo índice glicêmico por alimentos com altas cargas e índice glicêmico aumentou significativamente a glicemia e a demanda insulínica nas últimas décadas (OKAMOTO et al., 2015).

Essa inadequação da dieta, associada ao sedentarismo, são importantes fatores desencadeantes de doenças metabólicas, de tal maneira que a epidemia mundial de obesidade está diretamente relacionada ao número elevado de casos de diabetes mellitus (DM) tipo 2 (COELHO et al., 2009; MARIATH et al., 2007; PEREIRA et al., 2003).

Em 2000, a Organização Mundial da Saúde (OMS) estimou que o número de pessoas com sobrepeso chegaria a 2 bilhóes em 2030. A prevalência mundial da obesidade quase dobrou entre 1980 e 2008. Dados mais atuais mostram que cerca de 250 milhóes de pessoas estão nessa condição no mundo, sendo vários os países que sofrem com essa epidemia, inclusive o Brasil (OKAMOTO et al., 2015; WHO, 2010).

No Brasil, têm-se dados epidemiológicos da Vigilância de fatores de risco e proteção para doenças crônicas por Inquérito Telefônico (VIGITEL), levantamento anual realizado pelo Ministério da Saúde que ocorre desde 2006. No último VIGITEL, de 2014, foram monitorados 40.853 adultos com mais de 18 anos de idade, residentes nas 26 capitais dos estados e no Distrito Federal, sendo que $52,5 \%$ dos pesquisados estáo acima do peso. Quando esse inquérito iniciou, no ano de 2006, o índice era de 43\% (MINISTÉRIO 
DA SAÚDE, 2015). Justifica-se, portanto, a importância da Liga, visto que temas de alta prevalência farão parte da prática clínica dos membros ligantes.

Assim, este artigo objetiva apresentar a experiência que a LATGIDM teve em seu primeiro ano como projeto extensionista, tendo em vista a análise e compreensão de seu papel na formação dos acadêmicos, sua inserção como um importante agente de atenção à saúde no município e sua atuação na comunidade, por meio das atividades realizadas.

\section{Metodologia}

A LATGIDM é um projeto extensionista desenvolvido por alunos e professores de Medicina da Universidade Estadual de Ponta Grossa (UEPG) e a colaboraçáo da professora de Nutrição que atualmente presta serviço à UFPR, com início em 2015 . O projeto conta com a supervisão dos professores Marcos Ricardo da Silva Rodrigues, cirurgião geral e do aparelho digestivo, Cíbele Pereira Kopruszynski, professora atual de nutrição da UFPR, mas que durante o período da Liga acadêmica ainda não estava atuante nesta instituição, e Gianna Carla Alberti Schrut, endocrinologista clínica.

O projeto foi aberto a todos os níveis da graduação do curso de Medicina da UEPG. Os acadêmicos que tiveram interesse em participar da Liga e que compareceram à primeira reunião foram consagrados como membros efetivos. A Figura 1 demostra o logotipo da LATGIDM feita por um de seus membros.

\section{Figura 1: Logotipo da LATGIDM}

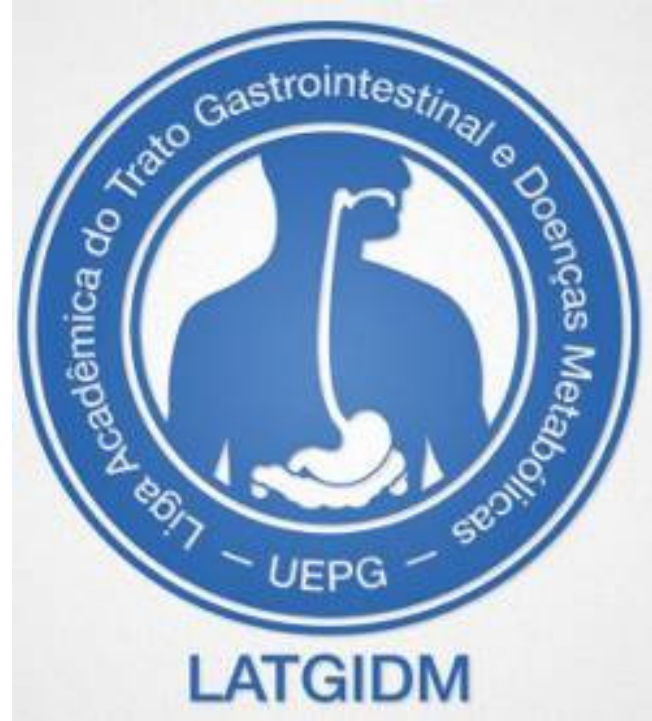

Fonte: os autores.

Os objetivos traçados foram o aprimoramento do conhecimento dos acadêmicos de Medicina da UEPG quanto às doenças do trato gastrointestinal e metabólicas (DM, tireoidopatias, obesidade, dislipidemia), tanto na teoria como na prática. Com isso, houve a inserção dos discentes na comunidade ou em ambulatórios do Hospital Regional, atendendo à demanda da populaçáo relacionada a essas doenças (OKAMOTO et al., 2015). 
Dessa forma, entende-se que um amplo conhecimento sobre a relação do consumo alimentar e suas consequências metabólicas auxiliam os alunos participantes da liga a desenvolverem açôes educativas voltadas à comunidade, orientaçôes para grupo de riscos, campanhas de promoção da saúde e prevenção primária relacionada às condiçóes patológicas mencionadas. Além disso, a LATGIDM objetiva a elaboração de projetos de pesquisa que divulguem o conhecimento produzido pelos seus integrantes e que valorizem o papel social da UEPG na comunidade na qual está inserida.

As reunióes científicas da Liga foram realizadas quinzenalmente, por um ano (período 2015-2016), em horário extracurricular, para que os acadêmicos pudessem participar, sem comprometimento da grade curricular básica. Os temas abordados foram ministrados pelos docentes e discentes (Quadro 1) com eventuais palestras de profissionais especialistas no tema pertinente. Foram discutidos temas de interesse geral, assim como discussão de artigos científicos específicos.

\section{Quadro 1: Assuntos abordados nos encontros da LATGIDM. Ponta Grossa, 2016}

\begin{tabular}{|c|}
\hline Aula inaugural e introdução à LATGIDM \\
\hline Aula sobre classes de alimentos \\
\hline Discussão sobre índice glicêmico e carga glicêmica dos alimentos \\
\hline Secreção entero-hormonal \\
\hline Discussão de artigo (SACKS et al., 2014) \\
\hline Inquéritos alimentares \\
\hline Fisiopatologia e bases moleculares da obesidade \\
\hline Fibras alimentares \\
\hline Microbiota intestinal \\
\hline Tratamento clínico da obesidade \\
\hline Transplante fecal \\
\hline Discussão de artigo (BUSE et al., 2009) \\
\hline Organização do Dia Mundial do diabetes mellitus \\
\hline Aula sobre hipertrigliceridemia \\
\hline Apresentação dos dados do Dia Mundial do diabetes \\
\hline Aula de encerramento do ciclo da Liga \\
\hline
\end{tabular}

A elaboração de material educativo sobre uma dieta equilibrada e hábitos saudáveis à população atendida no dia do diabetes também foi realizada pelos discentes e docentes. Pensando em uma abordagem didática e de leitura rápida, elaborou-se um material com a imagem de um prato saudável, de modo a facilitar a visualizaçấo das proporçôes recomendadas de cada nutriente. No verso, foram colocadas diversas opçôes de alimentos que podem substituir aqueles apresentados no prato saudável, com valor nutricional equivalente.

Por fim, os discentes e docentes dividiram-se em dois grupos iguais para realizar, em dois dias livres de atividades curriculares, exames de glicemia capilar, coleta de dados antropométricos, assim como uma anamnese que contemplava informaçôes relacionadas a dados familiares, pessoais e alimentares de uma população aleatória estudada. Ao final da entrevista, os pacientes eram orientados em relação a uma dieta balanceada e hábitos saudáveis, pelos alunos e profissionais, coordenadores da LATGIDM ali presentes (nutricionista, endocrinologista e cirurgião do aparelho digestivo). Tais atividades 
ocorreram em supermercados da Rede Condor, nos bairros Uvaranas e Nova Rússia, da cidade de Ponta Grossa - PR.

\section{Resultados e discussão}

Nesta seção, serão apresentados e discutidos os resultados obtidos pela LATGIDM a partir das açôes desenvolvidas. Com relação à área da pesquisa, os acadêmicos realizaram projetos de iniciação científica sob coordenação dos professores supervisores. Alguns trabalhos de conclusão de curso também foram idealizados a partir de discussão de temas das reuniōes científicas. Ressalta-se que uma revisão de literatura realizada por membros da Liga foi aceita para publicação em um periódico nacional (STUMPF et al., 2015). O Quadro 2 demonstra os temas de trabalhos oriundos da LATGIDM. Todos os projetos foram submetidos e aprovados pelo Comitê de Ética em Pesquisa.

\section{Quadro 2: Correlaçáo entre os temas das reunióes e os projetos de pesquisa. Ponta Grossa, 2016}

\begin{tabular}{|c|l|}
\hline $\begin{array}{c}\text { Tema da reunião } \\
\text { científica }\end{array}$ & Ideia do projeto de pesquisa \\
\hline \multirow{2}{*}{ Diabetes mellitus } & Avaliação da qualidade de vida e da prevalência de neuropatia diabética \\
\cline { 2 - 2 } & Avaliação do sono em pacientes com diabetes \\
\hline \multirow{2}{*}{ Cirurgia bariátrica } & Qualidade de vida antes e após a cirurgia bariátrica \\
\cline { 2 - 2 } & Análise da tolerância alimentar após a cirurgia bariátrica \\
\hline Entero-hormônios & $\begin{array}{l}\text { Alterações histológicas no trato gastrointestinal de ratos com diabetes } \\
\text { induzida }\end{array}$ \\
\hline
\end{tabular}

Em outro âmbito das atividades desenvolvidas, foram realizados acompanhamentos ambulatoriais, nos quais os integrantes da Liga, conforme disponibilidade, podiam atender pacientes sob supervisão de pelo menos um dos coordenadores da LATGIDM. O principal ambulatório acompanhado foi o do serviço de endocrinologia, que permitia ao acadêmico aprofundar o conhecimento teórico adquirido nas reuniôes da Liga.

A partir da experiência do acompanhamento e da inserção precoce dos acadêmicos no âmbito clínico, foram escritos dois resumos ao Congresso Brasileiro de Educação Médica (COBEM). Ressalta-se, dessa forma, o uso de atividades extracurriculares (como ligas) e o início precoce na prática clínica como oportunidades para a formação médica comprometida com a integração academia, serviço e comunidade. Vale ressaltar que, dentre 1.000 resumos enviados ao COBEM, somente 150 foram aceitos, sendo 7 da UEPG (dentro dos quais computam os enviados pela LATGIDM).

Os acadêmicos que participaram da LATGIDM ampliaram seus conhecimentos tanto teóricos quanto práticos, sendo notável a diferença da forma com que os conteúdos ministrados em sala de aula foram interpretados após as discussóes de artigos e casos clínicos, e o contato prático com o ambiente de atendimento à populaçáo.

A atuaçáo dos discentes nas ligas permitiu-lhes encontrar o espírito crítico, a criatividade, a capacidade de discernimento, de planejamento e açáo, e o compromisso ético para com a populaçáo. Fez ainda com que as teorias aprendidas em reuniôes 
científicas fossem utilizadas de maneira prática tanto para o desenvolvimento de habilidades semiológicas utilizadas nos ambulatórios como para o atendimento realizado na comunidade.

Assim, a presença do estudante, juntamente com os profissionais de saúde, permitiu a criação de um ambiente de trocas de conhecimento e prática. Sabe-se que o acadêmico que interage precocemente com os pacientes e com os profissionais adquire reconhecimento do ambiente de trabalho, da divisão de funçóes e auxilia no vínculo médico/paciente (PÊGO-FERNANDES et al., 2011; HAMAMOTO FILHO, 2011; VIERA et al., 2004).

Os integrantes da LATGIDM também desenvolveram noçóes de aprendizado em conjunto, de trabalho em equipe, de trocas de experiências e, ainda, a compreensão das necessidades dos pacientes, levando à formulação de orientaçóes em saúde mais específicas para cada pessoa atendida. Isso objetiva evitar a reproduçáo de modelos centrados na doença e no cientificismo que desconsidera o conhecimento empírico das pessoas.

Devido ao envolvimento dos discentes tanto na discussáo de artigo como na apresentação de aulas, participando ativamente da Liga acadêmica, mostrou-se que este espaço fornece condiçôes para o desenvolvimento de habilidades de comunicação com eficiência, respeito às opiniōes contrárias, discussão inteligente, além de compreensão de problemas e raciocínio para sua resolução (OKAMOTO et al., 2015).

Vários trabalhos que abordam temas como o tratado neste artigo apresentam resultados semelhantes. A maioria afirma que, quando os acadêmicos sáo inseridos dentro de um grupo que aborda determinado tema de grande interesse, em um ambiente construído e conduzido por eles próprios sob orientação, é possível uma grande aquisiçâao de aprendizado e experiência, desenvolvimento de raciocínio clínico-científico, ampliação do conhecimento, ao mesmo tempo em que se promove uma maior interaçáo com a comunidade. E, em longo prazo, estes acadêmicos se tornarão líderes de comunidade e formadores de opinião em suas diversas especialidades (FREITAS JÚNIOR et al., 2014; FERNANDES et al., 2011; MONTEIRO et al., 2008; HENNINGTON, 2005; JEZINE, 2004).

Como ação voltada para a comunidade, a LATGIDM participou do Dia Mundial do DM (24 de novembro), doença metabólica que afeta 415 milhóes de pessoas no mundo, segundo dados recentes da Federaçáo Internacional de Diabetes (IDF, 2015). Foram programados dois dias extracurriculares (14 e 21 de novembro, 2015), para realização de triagem na população.

Nesses dias, todos os integrantes da Liga participaram ativamente, realizando anamneses, aferindo pressão arterial, coletando dados antropométricos, além de realização de testes de glicemia sérica por meio de glicosímetro e fornecendo orientaçôes médicas e nutricionais direcionadas para cada pessoa atendida.

Ao final do atendimento, todos os pacientes eram orientados, através de demonstração de material educativo pré-elaborado pelos membros da LATGIDM (Figura 2), a adotarem uma dieta adequada, assim como foram estimuladas atitudes mais saudáveis, como prática de atividade física, cessar tabagismo, entre outras.

Percebeu-se grande adesão da comunidade que procurou o atendimento em supermercados da Rede Condor de Ponta Grossa (propaganda realizada pela Rede de Supermercados Condor - Figura 3). Na Figura 4, podem ser vistos alguns integrantes da Liga no segundo dia de atuação na comunidade. 
No futuro, os dados coletados seráo utilizados para desenvolvimento de outros trabalhos científicos, para relatos de experiência ou submissão de resumos em congressos.

\section{Figura 2: Material educativo elaborado pelos discentes da LATGIDM}

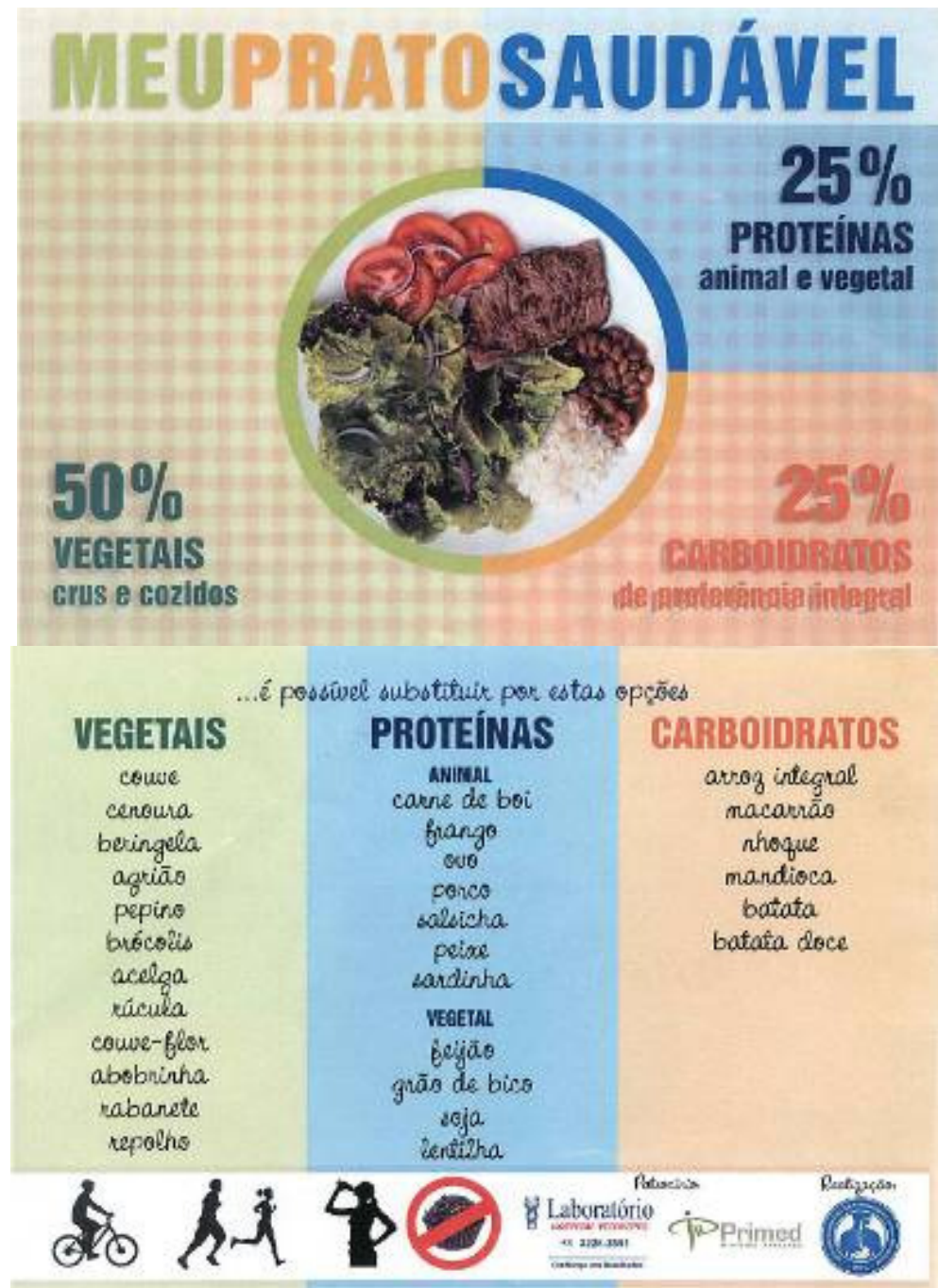

Fonte: Os autores.

Figura 3: Imagem veiculada em redes sociais pela Rede de Supermercados Condor de Ponta Grossa, sobre a atuaçáo da LATGIDM da UEPG

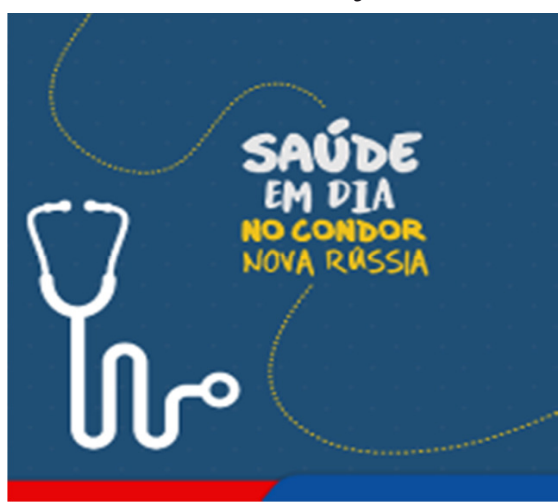

Disponível em: https://www.facebook.com/RedeCondor/photos/a.220283487995367.61271.212743445416038/107463936 2559771/?type=3\&theater. 


\section{Figura 4: Integrantes da LATGIDM da UEPG no segundo dia de atendimento à comunidade}

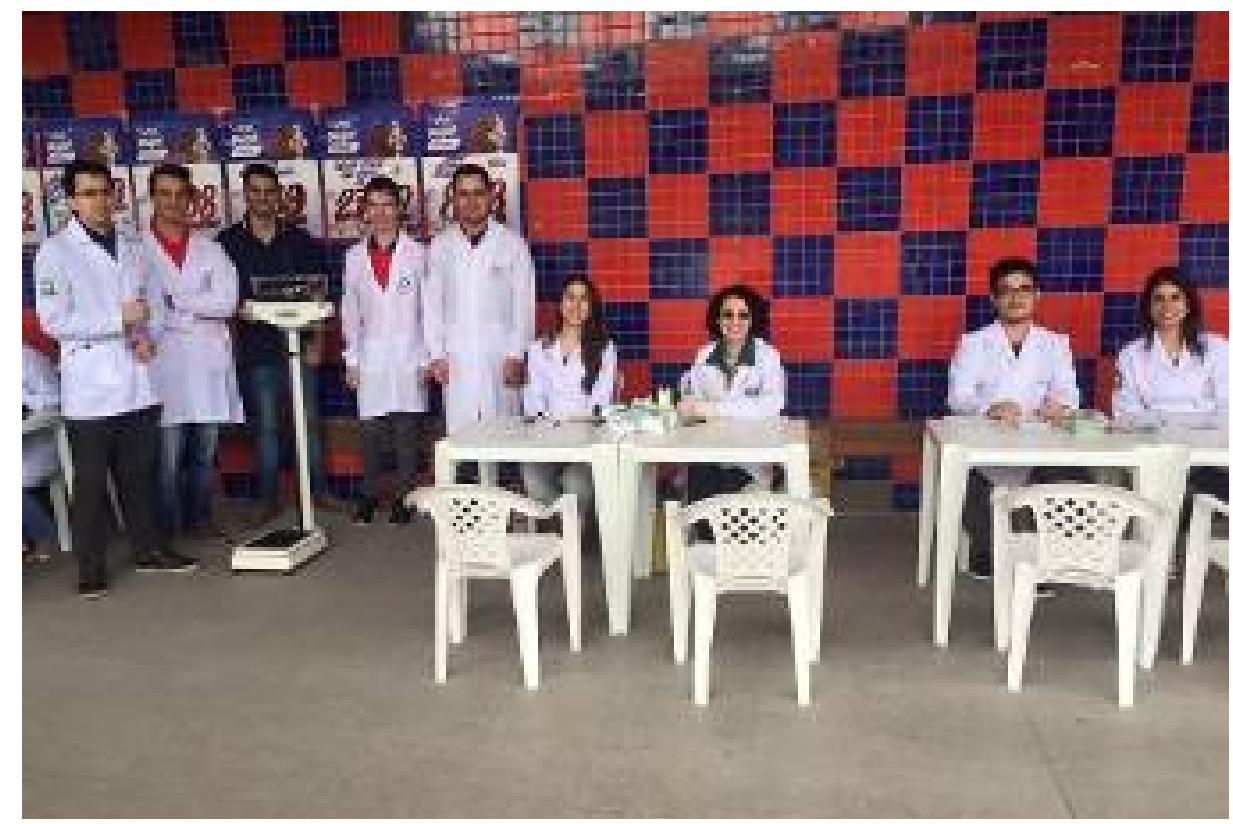

Fonte: Os autores.

\section{Conclusáo}

Tendo em vista a relevância do assunto tratado pela Liga, observa-se a importância desta para a formaçáo de novos profissionais e o ganho curricular que o discente adquire durante todo o processo de ensino, pesquisa e extensão. Soma-se a isso a importância e influência positiva que o projeto tem para a comunidade da região, melhorando e ampliando a prestação de serviço aos participantes das açóes extensionistas da LATGIDM.

Apesar de que um relato de experiência não permite a extrapolação de seus resultados para outras populaçôes, permite a indicação de estratégias e processos interessantes para valorizar a construção de competências nos estudantes que contribuem para sua formação crítica, reflexiva e cidadá.

Desta forma, a partir das atividades desenvolvidas e os resultados aqui apresentados, aponta-se que a LATGIDM oferece um papel importante na formaçáo médica, social, pessoal e o contato com a comunidade, atuando na reflexáo sobre os hábitos da população em geral que podem estar associados a doenças crônicas não transmissíveis, desencadeando maior índice de doenças metabólicas.

Assim, espera-se que as ligas médicas sejam modelos para que os acadêmicos possam adquirir conhecimentos práticos, com maior grau de satisfaçáo e de modo mais significativo. Que possam desenvolver suas habilidades e potenciais, assim como a capacidade crítica e reflexiva, de forma que isso reflita no atendimento à população. 


\section{Referências}

ANDRADE, A.S.; GARCIA, S.B.; PERES, C.M. Atividades extracurriculares: multiplicidade e diferenciação necessárias ao currículo. Revista Brasileira de Educação Médica, v.31, n.3, p.203-211, 2007.

BASTOS, M.L.S.; TRAJMAN, A.; TEIXEIRA, E.G.; SELIG, L.; BELO, M.T.C.T. O papel das ligas acadêmicas na formação profissional. Jornal Brasileiro de Pneumologia, v.38, n.6, 2012.

BUSE, J.B. et al. How Do We Define Cure of Diabetes? Diabetes Care, v.32, n.11, p.2133-2135, 2009.

COELHO, C.F.; BURINI, R.C. Atividade física para prevenção e tratamento das doenças crônicas não transmissíveis e da incapacidade funcional. Revista de Nutrição, v.22, n.6, p.937-946, 2009.

COSTA, A.P.; AFONSO, C.L.; DEMUNER, J.M.M.; MORAES, J.M.; PIRES, W.C. A importância da Liga acadêmica de queimaduras. Revista Brasileira de Queimaduras, v.8, n.3, p.101-105, 2009.

FERREIRA, D.A.V.; ARANHA, R.N.; SOUZA, M.H.F.O. Ligas Acadêmicas: uma proposta discente para ensino, pesquisa e extensão. Interagir: pensando a extensão, n.16, p.47-51, 2011.

FREITAS JÚNIOR, J.R.; PRZYBYCIEN, M.; TRENTINI, C.A.; CAMARGO, C.H.F. O papel da liga acadêmica de urgências e emergências cirúrgicas na formação médica. Revista Conexão UEPG, v.10, n.1, p.120-127, 2014.

HAMAMOTO FILHO, P.T.;VENDITTI, V.C.; OLIVEIRA, C.C.;VICENTINI, H.C.; SCHELLINI, S.A. Ligas acadêmicas de Medicina: extensão das ciências médicas à sociedade. Revista Ciência em Extensão, v.7, n.1, p.126-133, 2011.

HAMAMOTO FILHO, P.T. Ligas acadêmicas: motivações e críticas a propósito de um repensar necessário. Revista Brasileira de Educação Médica, v.35, n.4, p.535-543, 2011.

HAMAMOTO FILHO, P.T. et al. Normatização da abertura de ligas acadêmicas: a experiência da Faculdade de Medicina de Botucatu. Revista Brasileira de Educação Médica, v.34, n.1, p.160-167, 2010.

HENNINGTON, E.A. Acolhimento Como Prática Interdisciplinar Num Programa de Extensão Universitária. Cadernos de Saúde Pública, v.21, n.1, p.256-265, 2005.

INTERNATIONAL DIABETES FEDERATION. IDF Diabetes Atlas - 7th Edition, 2015. Disponível em: <http://www.diabetesatlas.org/>. Acesso em: 25/03/2016.

JEZINE, E. As Práticas Curriculares e a Extensão Universitária. In: CONGRESSO BRASILEIRO DE EXTENSÃO UNIVERSITÁRIA, 2., 2004, Belo Horizonte. Anais... Belo Horizonte: ABEU, 2004.

MARIATH, A.B. et al. Obesidade e fatores de risco para o desenvolvimento de doenças crônicas não transmissíveis entre usuários de unidade de alimentação e nutrição. Cadernos de Saúde Pública, v.23, n.4, p.897-905, 2007. 
MELLO, V.D.; LAAKSONEN, D.E. Fibras na dieta: tendências atuais e benefícios à saúde na síndrome metabólica e no diabetes melito tipo 2. Arquivos Brasileiros de Endocrinologia e Metabologia, v.53, n.5, p.509-518, 2009.

MINISTÉRIO DA SAÚDE (MS). Vigitel Brasil 2014 Saúde Suplementar: vigilância de fatores de risco e proteção para doenças crônicas por inquérito telefônico. Ministério da Saúde, Agência Nacional de Saúde Suplementar. Disponível em: http://www.ans.gov.br/images/stories/Materiais_para_pesquisa/Materiais_ por_assunto/2015_vigitel.pdf. Acesso em: 12 abr. 2016.

MONTEIRO, L.L.F.; CUNHA, M.S.; OLIVEIRA, W.L.; BANDEIRA, N.G.; MENEZES, J.V. Ligas acadêmicas: O que há de positivo? Experiência de implantação da Liga Baiana de Cirurgia Plástica. Revista Brasileira de Cirurgia Plástica, v.23, n.3, p.158-161, 2008.

NEVES, F.B.C.S. et al. Inquérito nacional sobre as ligas acadêmicas de Medicina Intensiva. Revista Brasileira de Terapia Intensiva, v.20, n.1, p.43-48, 2008.

OKAMOTO, J.M., STUMPF, M.A.M., VEIGA, R.B., SCHURT, G.C.A., RODRIGUES, M.R.S. Apresentação da liga acadêmica do trato gastrointestinal e doenças metabólicas. In: CONEX - CONVERSANDO SOBRE EXTENSÃO, 13., Ponta Grossa, 2015. Anais... Ponta Grossa: UEPG, 2015.

PÊGO-FERNANDES, P.M.; MARIANI, A.W. O ensino médico além da graduação: ligas acadêmicas. Diagnóstico e Tratamento, v.16, n.2, p.50-51, 2011.

PEREIRA, L.O.; FRANCISCHI, R.P.; LANCHA JR, A.H. Obesidade: hábitos nutricionais, sedentarismo e resistência à insulina. Arquivos Brasileiros de Endocrinologia e Metabologia, São Paulo, v. 47, n. 2, p. 111-127, 2003.

SACKS, F.M. et al. Effects of High vs Low Glycemic Index of Dietary Carbohydrate on Cardiovascular Disease Risk Factors and Insulin Sensitivity The OmniCarb Randomized Clinical Trial. The Journal of the American Medical Association, v.312, n.23, p.2531-2541, 2014.

SANTANA, A.C.D.A. Ligas acadêmicas estudantis: o mérito e a realidade. Medicina Ribeirão Preto, v.45, n.1, p.96-98, 2012.

STUMPF, M.A.M; RODRIGUES, M.R.S.; KLUTHCOVSKY, A.C.G.C.; TRAVALINI, F.; MILLEO, F.Q.M. Análise da tolerância alimentar em pacientes submetidos à cirurgia bariátrica através do questionário Quality of Alimentation. Arquivos Brasileiros de Cirurgia Digestiva, v.28, p.79-83, 2015.

VIEIRA, E.M. et al. O que eles fazem depois da aula? As atividades extracurriculares dos alunos de ciências médicas da FMRP-USP. Medicina Ribeirão Preto, v.37, n.1, p.84-90, 2004.

WORLD HEALTH ORGANIZATION (WHO). Obesity: Data and Statistics, 2010. Disponível em:<http://www.euro.who.int/en/health-topics/ noncommunicablediseases/obesity/data-and-statistics $>$. Acesso em: 25/03/2016. 